\title{
(2) OPEN ACCESS \\ JAK selectivity and the implications for clinical inhibition of pharmacodynamic cytokine signalling by filgotinib, upadacitinib, tofacitinib and baricitinib
}

\author{
Paqui G Traves, ${ }^{1}$ Bernard Murray, ${ }^{2}$ Federico Campigotto, ${ }^{3}$ René Galien, ${ }^{4}$ Amy Meng, ${ }^{5}$ \\ Julie A Di Paolo ${ }^{6}$
}

\begin{abstract}
Handling editor Josef S Smolen

- Additional material is published online only. To view please visit the journal online (http://dx.doi.org/10.1136/ annrheumdis-2020-219012)
\end{abstract}

'Inflammation Biology, Gilead Sciences, Foster City, California, USA

${ }^{2}$ Drug Metabolism, Gilead Sciences, Foster City, California, USA

${ }^{3}$ Biology Core Support, Gilead Sciences, Foster City, California, USA

${ }^{4}$ Translational Research, Galapagos SASU, Romainville, France

${ }^{5}$ Clinical Pharmacology, Gilead Sciences, Foster City, California USA

${ }^{6}$ External Innovation, Gilead Sciences, Foster City, California, USA

\section{Correspondence to}

Dr Julie A Di Paolo;

julie.dipaolo@gilead.com

Received 1 September 2020 Revised 12 January 2021 Accepted 13 January 2021 Published Online First 19 March 2021

Check for updates

\section{(c) Author(s) (or their} employer(s)) 2021. Re-use permitted under CC BY-NC. No commercial re-use. See rights and permissions. Published by BMJ.

To cite: Traves $P G$

Murray $B$, Campigotto $F$,

et al. Ann Rheum Dis

2021:80:865-875.

\section{ABSTRACT}

Objective Janus kinase inhibitors (JAKinibs) are efficacious in rheumatoid arthritis (RA) with variable reported rates of adverse events, potentially related to differential JAK family member selectivity. Filgotinib was compared with baricitinib, tofacitinib and upadacitinib to elucidate the pharmacological basis underlying its clinical efficacy and safety.

Methods In vitro JAKinib inhibition of signal transducer and activator of transcription phosphorylation (pSTAT) was measured by flow cytometry in peripheral blood mononuclear cells and whole blood from healthy donors and patients with RA following cytokine stimulation of distinct JAK/STAT pathways. The average daily PSTAT and time above $50 \%$ inhibition were calculated at clinical plasma drug exposures in immune cells. The translation of these measures was evaluated in ex vivo-stimulated assays in phase 1 healthy volunteers.

Results JAKinib potencies depended on cytokine stimulus, PSTAT readout and cell type. JAK1-dependent pathways (interferon (IFN)o/pSTAT5, interleukin (IL)-6/ pSTAT1) were among the most potently inhibited by all JAKinibs in healthy and RA blood, with filgotinib exhibiting the greatest selectivity for JAK1 pathways. Filgotinib (200 mg once daily) had calculated average daily target inhibition for IFN $\alpha / p S T A T 5$ and IL-6/pSTAT1 that was equivalent to tofacitinib ( $5 \mathrm{mg}$ two times per day), upadacitinib (15 mg once daily) and baricitinib (4 mg once daily), with the least average daily inhibition for the JAK2-dependent and JAK3-dependent pathways including IL-2, IL-15, IL-4 (JAK1/JAK3), IFN $\gamma$ (JAK1/ JAK2), granulocyte colony stimulating factor, IL-12, IL-23 (JAK2/tyrosine kinase 2) and granulocyte-macrophage colony-stimulating factor (JAK2/JAK2). Ex vivo pharmacodynamic data from phase 1 healthy volunteers clinically confirmed JAK1 selectivity of filgotinib.

Conclusion Filgotinib inhibited JAK1-mediated signalling similarly to other JAKinibs, but with less inhibition of JAK2-dependent and JAK3-dependent pathways, providing a mechanistic rationale for its apparently differentiated efficacy:safety profile.

\section{INTRODUCTION}

The Janus kinase (JAK) and signal transducers and activators of transcription (STAT) proteins constitute the JAK-STAT pathways, which are essential to immune regulation. Signalling through the pathways is initiated when a cytokine binds its cell-surface receptor, activating receptor-associated JAKs and phosphorylating STAT proteins. The

\section{Key messages}

What is already known about this subject?

- Janus kinase (JAK) inhibitors (JAKinibs) have emerged as an important new class of oral therapy for the treatment of rheumatoid arthritis (RA).

- Despite showing similar clinical efficacy, the reported incidence rates of some adverse events of special interest vary among the JAKinibs.

- The relationship between JAK isoform selectivity and inhibition of distinct cytokine responses at clinical plasma exposures of these JAKinibs could provide a mechanistic basis for their relative efficacy and safety profiles.

What does this study add?

- This study is the first to combine in vitro inhibition of cytokine responses in whole blood with clinical pharmacokinetics of filgotinib, baricitinib, tofacitinib and upadacitinib to model daily cytokine-mediated pharmacodynamic profiles in healthy individuals and patients with RA.

- These data demonstrate that, compared with upadacitinib, tofacitinib and baricitinib, filgotinib had similar calculated daily average inhibition of JAK1-dependent pathways activated by interleukin 6 and interferon $\alpha$, but the least inhibition of JAK2-dependent and JAK3-dependent signalling.

- The observed inhibition of JAKinibs on cytokine signalling was highly nuanced, and it was observed to be dependent on cytokine stimulus, STAT (signal transducers and activators of transcription) substrate and cell type, indicating that the differential cytokine profiles may provide a mechanistic rationale for reported efficacy and safety.

STATs dimerise and migrate to the nucleus to induce and/or maintain immune responses via transcriptional regulation.

The JAK family consists of JAK1, JAK2, JAK3 and tyrosine kinase 2 (TYK2). They bind intracellularly to dimeric cytokine receptor chains in pairs that vary by receptor. ${ }^{12}$ Depending on which JAK is activated, the functional effects vary. Specific JAK pairs are implicated in diverse functions that regulate inflammation, ${ }^{3}$ haematopoiesis ${ }^{2}$ 4-8 and 


\section{Key messages}

How might this impact on clinical practice or future developments?

- This study demonstrates that JAKinibs have unique, differential effects on specific cytokine signalling pathways and supports that preferential JAK1 activity is sufficient to drive RA efficacy, while JAK2 and JAK3 inhibition may increase the incidence of adverse events of special interest.

- These data can be used to build mechanistic correlations between cytokine inhibition and rates of adverse events observed with real-world JAKinib use.

immune homeostasis (figure 1). ${ }^{9-14}$ The JAK-STAT pathways also play essential roles in immune-mediated pathology, including rheumatoid arthritis (RA). ${ }^{6}$ Uncontrolled cytokine expression drives chronic inflammation; inadequately treated, this leads to systemic illness, joint destruction and deformity that characterise RA.

JAK inhibitors (JAKinibs) have emerged as an important new class of oral therapy in RA. ${ }^{16}$ Baricitinib, ${ }^{16}$ tofacitinib $^{17} 18$ and upadacitinib ${ }^{19}$ are currently approved in the USA, European Union, Japan and other countries. Filgotinib is a novel JAKinib and has recently been approved in the European Union

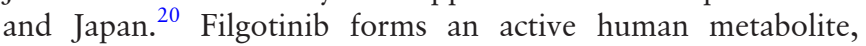
GS-829845/G254445, ${ }^{21}$ that contributes to its pharmacological activity. ${ }^{22}$ Studies suggest that JAK1 inhibition might be largely responsible for the efficacy of JAKinibs in RA. ${ }^{23}{ }^{24}$ Biochemically, all these JAKinibs show the greatest potency at inhibiting JAK1, with varying levels of selectivity for other JAK isoforms. ${ }^{21} 25-29$ While these inhibitors have similar efficacy in patients with RA, reported rates of adverse events (AEs) differ, including: increased incidences of herpes zoster (HZ), serious infections, venous thromboembolism, decreased natural killer (NK) cell numbers, thrombocytopaenia and anaemia (online supplemental table 1). ${ }^{16-20}$ Genetic evidence has linked JAK2 to erythropoiesis and thrombopoiesis, JAK3 to lymphocyte proliferation and immune homeostasis, and TYK2 to antiviral responses. ${ }^{6} 3031$ Therefore, differences in JAKinib selectivity for cytokine signalling via distinct JAK pairs may provide a mechanistic rationale for reported differences in safety profiles.

The objective of this study was to calculate the daily clinical pharmacodynamic (PD) inhibition profiles of cytokine signalling for filgotinib, baricitinib, tofacitinib and upadacitinib to compare their inhibition and selectivity. To accomplish this, we used a combination of in vitro cellular cytokine assays in human peripheral blood mononuclear cells (PBMCs) and whole blood (WB), coupling these results with the clinical RA plasma exposures of each JAKinib. Furthermore, we confirmed these results through ex vivo PD data obtained from blood samples from phase 1 healthy volunteers administered filgotinib.

\section{METHODS}

Detailed experimental procedures are depicted in figure 2 and described in the online supplemental materials.

\section{RESULTS}

JAK isoforms differentially contribute to JAK-STAT pathway activity

JAKinibs showed dose-dependent inhibition of cytokinestimulated phosphorylated STAT (pSTAT) levels in CD4+ and CD8 + T-cells, monocytes, NK cells, neutrophils and B-cells in human WB (tables 1 and 2, online supplemental figures 1-4) and PBMCs (online supplemental tables 2 and 3). For a given cell type, STAT substrate and cytokine stimulus, potency differences were observed between JAKinibs. The potencies measured in WB were consistently weaker than those assessed in PBMCs (tables 1
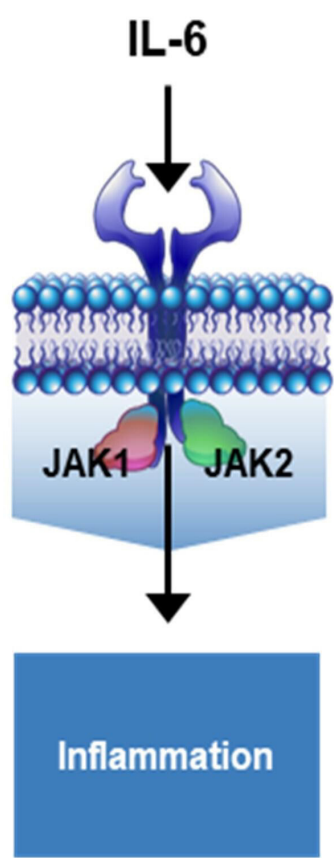
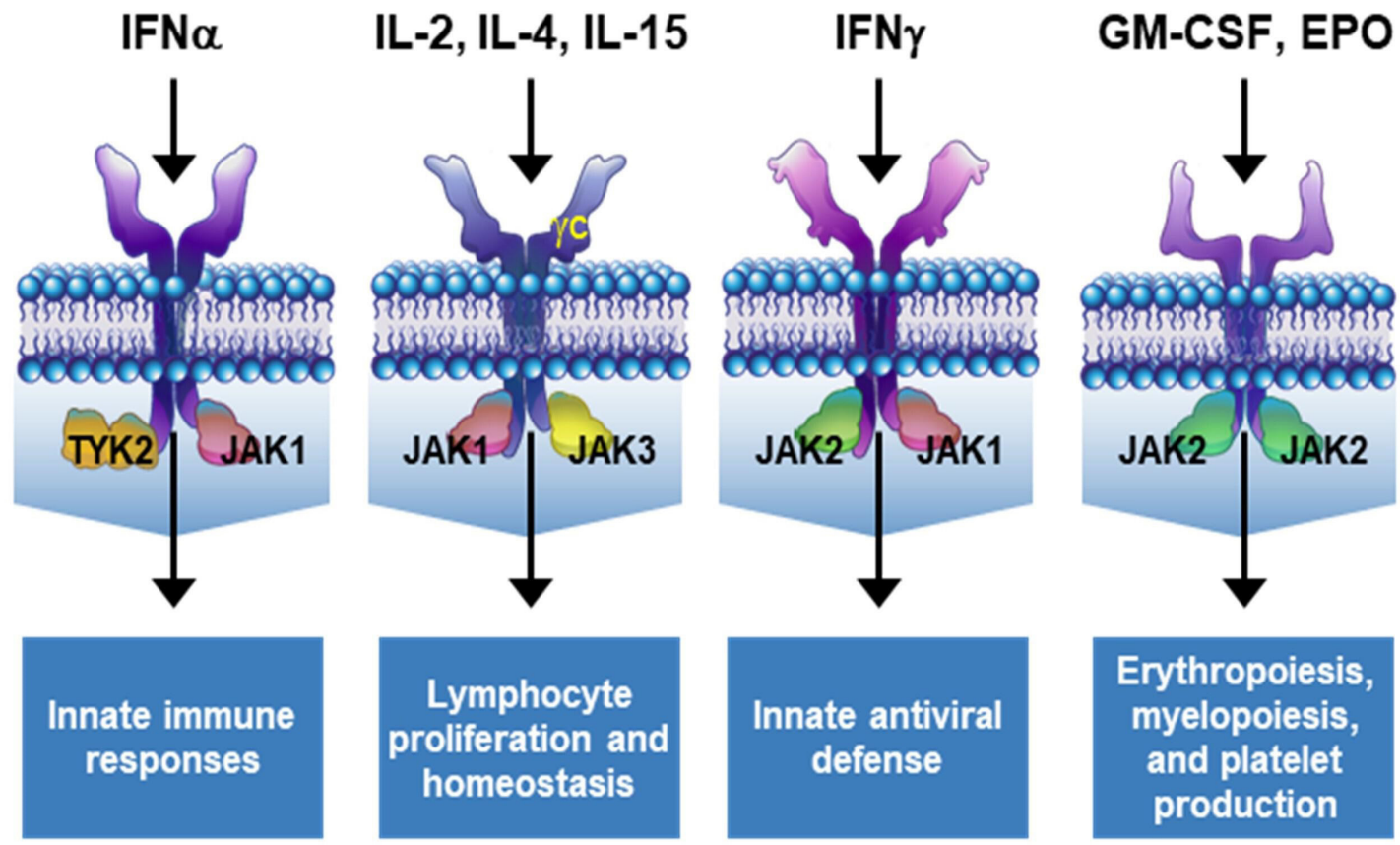

Figure 1 Cytokine receptors are associated with distinct JAK pairing patterns. The JAK isoforms involved in each pathway vary according to the specific cytokine receptor and dictate downstream outcomes. Figure adapted from Winthrop. ${ }^{49}$ EPO, erythropoietin; GM-CSF, granulocyte-macrophage colony-stimulating factor; IFN, interferon; IL, interleukin; JAK, Janus kinase; TYK2, tyrosine kinase 2. 


\section{A. Experimental procedure}

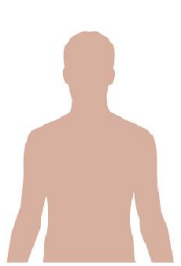

HD/RA

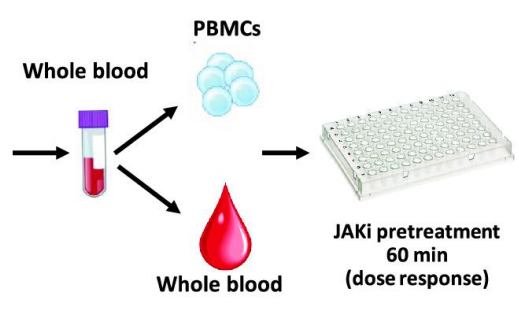

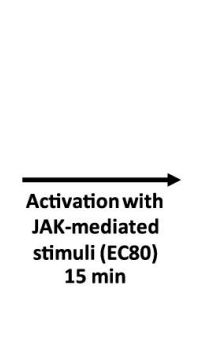

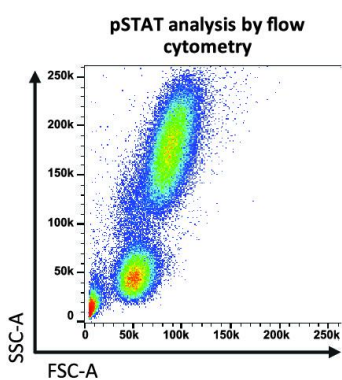

\section{B. Data analysis procedure}
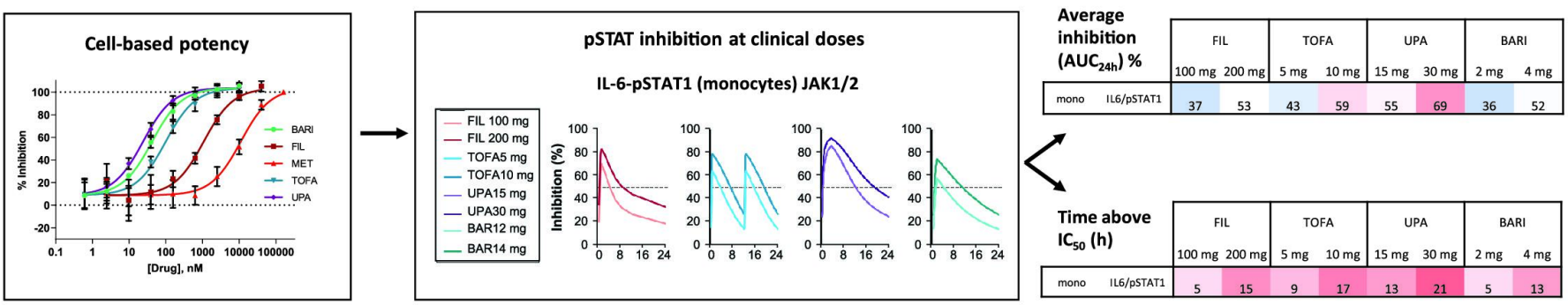

Figure 2 (A) Experimental design and (B) data analysis procedures. Detailed methods are included in online supplemental materials. AUC-24h,

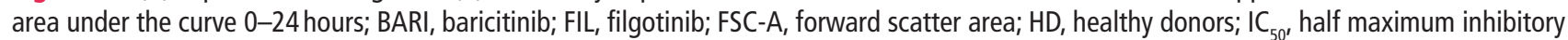
concentration; IL, interleukin; JAK, Janus kinase; JAKi, JAK inhibitor; MET, major metabolite of filgotinib (GS-829845); mono, monocytes; PBMCs, peripheral blood mononuclear cells; pSTAT, phosphorylated signal transducer and activator of transcription; RA, rheumatoid arthritis; SSC-A, side scatter area;TOFA, tofacitinib; UPA, upadacitinib.

and 2, online supplemental tables 2 and 3). This discrepancy was accounted for by compound plasma binding (online supplemental figure 5).

Cell type impacted the measured JAKinib potency to inhibit cytokine signalling. For example, comparing WB CD4+ cells and monocytes, there was an approximately threefold difference for the JAK1/TYK2-dependent interferon (IFN) $\alpha$-stimulated pSTAT1 for each JAKinib $(p<0.001)$, whereas for
B and NK cells, the potencies were comparable (tables 1 and 2, online supplemental table 4). For each cytokine evaluated, cell type affected JAKinib potencies with varying magnitudes of significance.

JAKinib potencies were dependent on the STAT substrate phosphorylated in response to a given stimulus. In healthy donors, inhibition was consistently 7 -fold to 11 -fold greater for JAK1/JAK2-driven interleukin (IL)-6/pSTAT1 than for

Table 1 JAKinib IC 50 values in CD4+ T-cells, monocytes and NK cells from whole blood assays

\begin{tabular}{|c|c|c|c|c|c|c|c|c|c|c|c|c|c|c|c|}
\hline \multirow[b]{3}{*}{ Stimulation/pSTAT } & \multicolumn{5}{|c|}{ CD4+ T-cells } & \multicolumn{5}{|c|}{ Monocytes } & \multicolumn{5}{|c|}{ NK cells } \\
\hline & BARI & FIL & MET & TOFA & UPA & BARI & FIL & MET & TOFA & UPA & BARI & FIL & MET & TOFA & UPA \\
\hline & \multicolumn{15}{|c|}{$\mathrm{IC}_{50}, \mathrm{nM}$} \\
\hline \multicolumn{16}{|c|}{ JAK2/JAK2 or JAK2/TYK2-dependent cytokines } \\
\hline G-CSF/pSTAT3 & & & NS & & & 81 & 4977 & 50215 & 292 & 81 & & & NS & & \\
\hline GM-CSF/pSTAT5 & & & NS & & & 127 & 9916 & 102910 & 510 & 74 & & & NS & & \\
\hline IL-12/pSTAT4 & & & NS & & & NS & & & & & 269 & 10351 & 221777 & 1216 & 364 \\
\hline \multicolumn{16}{|c|}{ JAK1/JAK2/TYK2-dependent cytokines } \\
\hline IFN $\alpha / p S T A T 1$ & 50 & 1096 & 17161 & 98 & 30 & 192 & 4560 & 91078 & 393 & 83 & 131 & 2440 & 41161 & 256 & 117 \\
\hline IFNo/pSTAT3 & 39 & 871 & 12644 & 80 & 24 & 40 & 991 & 15793 & 86 & 17 & 30 & 675 & 8620 & 60 & 25 \\
\hline IFN $\alpha / p S T A T 5$ & 28 & 638 & 9587 & 49 & 17 & 25 & 613 & 9518 & 51 & 11 & 34 & 507 & 7484 & 50 & 26 \\
\hline IFN $\gamma / \mathrm{pSTAT1}$ & & & NS & & & 74 & 4138 & 62374 & 228 & 58 & & & NS & & \\
\hline IL-6/pSTAT1 & 29 & 783 & 5637 & 63 & 27 & 39 & 1011 & 10019 & 84 & 22 & & & NS & & \\
\hline IL-6/pSTAT3 & 274 & 5435 & 62680 & 644 & 225 & 161 & 3527 & 49109 & 368 & 100 & & & NS & & \\
\hline \multicolumn{16}{|c|}{ JAK1/JAK3-dependent cytokines } \\
\hline IL-2/pSTAT5 & 40 & 988 & 14079 & 40 & 21 & & & NS & & & 80 & 2153 & 23824 & 87 & 56 \\
\hline IL-4/pSTAT6 & 73 & 1458 & 39420 & 77 & 36 & 53 & 1337 & 36537 & 116 & 32 & 40 & 869 & 20984 & 45 & 25 \\
\hline IL-15/pSTAT5 & 38 & 967 & 14326 & 39 & 21 & & & NS & & & 83 & 2044 & 27540 & 93 & 58 \\
\hline
\end{tabular}

G-CSF, GM-CSF, IFN $\alpha$, IFN $\gamma$, IL-6, IL-2, IL-4 and IL-15 reported IC 50 values are based on the average of duplicates from 7-10 healthy volunteer whole blood.

BARI, baricitinib; FIL, filgotinib; G-CSF, granulocyte colony stimulating factor; GM-CSF, granulocyte-macrophage colony stimulating factor; IC $\mathrm{C}_{50}$, half maximum inhibitory concentration; IFN, interferon; IL, interleukin; JAK, Janus kinase; JAKinib, Janus kinase inhibitor; MET, major metabolite of filgotinib (GS-829845); NK, natural killer cell; NS, not sampled; pSTAT, phosphorylated signal transducer and activator of transcription; TOFA, tofacitinib; TYK2, tyrosine kinase 2; UPA, upadacitinib. 
Table 2 JAKinib IC $_{50}$ values in B-cells, neutrophils and CD8+ T-cells from whole blood assays

\begin{tabular}{|c|c|c|c|c|c|c|c|c|c|c|c|c|c|c|c|}
\hline \multirow[b]{3}{*}{ Stimulation/pSTAT } & \multicolumn{5}{|c|}{ B-cells } & \multicolumn{5}{|c|}{ Neutrophils } & \multicolumn{5}{|c|}{ CD8+ T-cells } \\
\hline & BARI & FIL & MET & TOFA & UPA & BARI & FIL & MET & TOFA & UPA & BARI & FIL & MET & TOFA & UPA \\
\hline & \multicolumn{15}{|c|}{$\mathrm{IC}_{50}, \mathrm{nM}$} \\
\hline \multicolumn{16}{|c|}{ JAK2/JAK2 or JAK2/TYK2-dependent cytokines } \\
\hline G-CSF/pSTAT3 & & & NS & & & 404 & 16717 & 158111 & 1245 & 369 & & & NS & & \\
\hline GM-CSF/pSTAT5 & & & NS & & & 66 & 3436 & 40925 & 143 & 21 & & & NS & & \\
\hline IL-23/pSTAT3* & & & NS & & & NS & & & & & 210 & 15040 & 138638 & 970 & 368 \\
\hline \multicolumn{16}{|c|}{ JAK1/JAK2/TYK2-dependent cytokines } \\
\hline IFN $\alpha / p S T A T 1$ & 156 & 2957 & 54052 & 322 & 112 & & & NS & & & 80 & 1809 & 27730 & 163 & 56 \\
\hline IFNo/pSTAT3 & 28 & 588 & 9777 & 62 & 20 & 47 & 833 & 13438 & 66 & 18 & 35 & 795 & 10617 & 73 & 23 \\
\hline IFN $\alpha / p S T A T 5$ & 23 & 436 & 7524 & 44 & 16 & & & NS & & & 27 & 607 & 8231 & 46 & 17 \\
\hline IFN $\gamma / \mathrm{pSTAT1}$ & 22 & 900 & 12820 & 61 & 21 & 78 & 3137 & 42649 & 152 & 46 & & & NS & & \\
\hline \multicolumn{16}{|c|}{ JAK1/JAK3-dependent cytokines } \\
\hline IL-2/pSTAT5 & & & NS & & & & & NS & & & 32 & 809 & 11046 & 33 & 21 \\
\hline IL-4/pSTAT6 & 295 & 6426 & 164309 & 356 & 162 & 71 & 1200 & 37069 & 106 & 37 & 47 & 1022 & 25900 & 51 & 26 \\
\hline IL-15/pSTAT5 & & & NS & & & & & NS & & & 56 & 1459 & 19958 & 56 & 32 \\
\hline
\end{tabular}

G-CSF, GM-CSF, IFN $\alpha$, IFN $\gamma$, IL-2, IL-4 and IL-15 reported IC s0 $_{50}$ values are based on the average of duplicates from $6-10$ healthy volunteer whole blood.

*Memory CD8+ T-cells.

BARI, baricitinib; FIL, filgotinib; G-CSF, granulocyte colony stimulating factor; GM-CSF, granulocyte-macrophage colony-stimulating factor; $\mathrm{IC}_{50}$, half maximum inhibitory concentration; IFN, interferon; IL, interleukin; JAK, Janus kinase; JAKinib, Janus kinase inhibitor; MET, major metabolite of filgotinib (GS-829845); NS, not sampled; pSTAT, phosphorylated signal transducer and activator of transcription; TOFA, tofacitinib; TYK2, tyrosine kinase 2; UPA, upadacitinib.

IL-6/pSTAT3 $(\mathrm{p}<0.001)$, potentially implicating that JAK1 is predominantly mediating phosphorylation of STAT1 and JAK2 is mediating STAT3 (tables 1 and 2, online supplemental table 5). Similarly, JAKinib inhibition of JAK1/TYK2-mediated IFN $\alpha$ driven pSTAT5 and pSTAT3 was more potent than pSTAT1, again potentially demonstrating the reported reliance on TYK2 for regulating STAT1 phosphorylation. ${ }^{32}$

JAKinibs showed differences in inhibition of different cytokines using the same JAK pair. Filgotinib showed an approximately fourfold potency shift for JAK1/JAK2-dependent IL-6/ pSTAT1 compared with IFN $\gamma /$ pSTAT1 in monocytes $(\mathrm{p}<0.001)$ (table 1, online supplemental table 6). For other JAKinibs, potency differences were observed, although with less magnitude. These data highlight that cytokine receptors using identical JAK pairs may have differential reliance on a JAK isoform for mediating signalling.

\section{JAKinibs demonstrate distinct cellular selectivity}

Selectivity between JAKinibs was analysed by measuring inhibition of cytokine signalling via each JAK pair in WB monocytes or NK cells (table 1). JAKinibs most potently inhibited the JAK1/ TYK2-dependent IFN $\alpha / p S T A T 5$. Measured half maximum inhibitory concentration $\left(\mathrm{IC}_{50}\right)$ values for other cytokine responses were normalised to this value to control for intrinsic JAKinib potency differences (figure 3). All JAKinibs showed selectivity within approximately fivefold on JAK1-dependent pathways including IL-6 (JAK1/JAK2) and IL-15 (JAK1/JAK3). Conversely, JAKinibs showed differential selectivity against JAK2-mediated pathways. Filgotinib, GS-829845, tofacitinib and upadacitinib showed more than fivefold selectivity versus JAK2-dependent granulocyte colony stimulating factor (G-CSF)driven or granulocyte-macrophage colony-stimulating factor (GM-CSF)-driven signalling, with filgotinib demonstrating the greatest selectivity against JAK2 compared with JAK1 (IFN $\alpha$ ). Baricitinib showed lower JAK1 selectivity $(\leq 5.1$-fold for JAK1 versus non-JAK1 pathways; figure 3 ).
JAKinibs show differentiated JAK1-selective pharmacological profiles at clinical plasma exposures

Representative cytokine/pSTAT pathway inhibition profiles were modelled over a 24 -hour period at clinically relevant drug

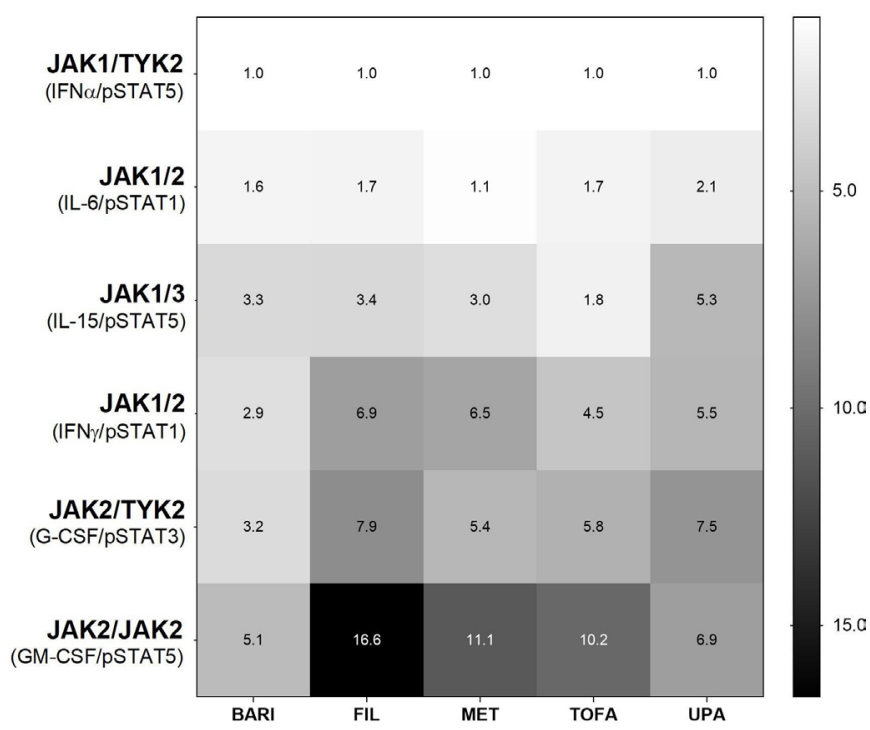

Figure 3 Distinct JAKinib cellular selectivity for JAK heterodimeric cytokine signalling. Mean fold selectivity for each JAK-dimer pair normalised to inhibition of JAK1/TYK2 pathway (IFN $\alpha / p S T A T 5$ in monocytes). Cytokine/pSTAT pairs: JAK1/TYK2 (IFN $\alpha / p S T A T 5$ in monocytes); JAK1/2 (IL-6/pSTAT1 in monocytes); JAK1/3 (IL-15/pSTAT5 in NK cells); JAK1/2 (IFN $\gamma /$ pSTAT1 in monocytes); JAK2/TYK2 (G-CSF/ pSTAT3 in monocytes); and JAK2/2 (GM-CSF/pSTAT5 in monocytes). BARI, baricitinib; FIL, filgotinib; G-CSF, granulocyte-colony stimulating factor; GM-CSF, granulocyte macrophage colony stimulating factor; IFN, interferon; IL, interleukin; JAK, Janus kinase; JAKinib, JAK inhibitor; MET, major metabolite of filgotinib (GS-829845); NK, natural killer; pSTAT, phosphorylated signal transducer and activator of transcription; TOFA, tofacitinib; TYK2, tyrosine kinase 2; UPA, upadacitinib. 
plasma concentrations in patients with RA at low and high JAKinib doses evaluated in the phase 3 clinical programmes (figure 4). ${ }^{33-35}$

The predicted average inhibition and time above 50\% inhibition $\left(\mathrm{IC}_{50}\right.$ coverage) of the JAK1/JAK2-mediated IL-6/pSTAT1 (figure 4A) and JAK1/TYK2-mediated IFNo/pSTAT5 (figure 4B) are similar among JAKinibs at doses reported to have similar overall American College of Rheumatology efficacy responses (filgotinib $200 \mathrm{mg}$ once daily; baricitinib $4 \mathrm{mg}$ once daily; tofacitinib $5 \mathrm{mg}$ two times per day; and upadacitinib $15 \mathrm{mg}$ once daily) (online supplemental table 1). For IL-6/pSTAT1, all JAKinibs showed similar inhibition at therapeutic doses. For IFNo/ pSTAT5, filgotinib had comparable daily inhibition as baricitinib and tofacitinib, and slightly less than upadacitinib. Some differences were noted in the time above $50 \%$ inhibition among JAKinibs. Thus, globally, the four JAKinibs demonstrate comparable $\mathrm{IC}_{50}$ coverage and percentage of pSTAT inhibition at therapeutic doses for these 2 JAK1-driven pathways.

On other JAK1/JAK2-dependent signalling pathways (IFN- $\gamma$-induced pSTAT1) in representative cell types, filgotinib $200 \mathrm{mg}$ displayed comparable or lower inhibition and time above $50 \%$ inhibition compared with baricitinib $4 \mathrm{mg}$, tofacitinib $5 \mathrm{mg}$ and upadacitinib $15 \mathrm{mg}$ (figure 4C,D). At these same doses, the average daily inhibition of JAK1/JAK3-dependent IL-4/pSTAT6 with filgotinib and baricitinib was comparable, but significantly lower than with tofacitinib and upadacitinib $(\mathrm{p}<0.001)$. Time above $50 \%$ inhibition was $\geq 3$ times longer for tofacitinib and upadacitinib compared with filgotinib $200 \mathrm{mg}$ $(\mathrm{p}<0.001)$ (figure 4D). Similarly, the average daily inhibition and time above $50 \%$ inhibition of IFN $\gamma /$ pSTAT1 were lowest for filgotinib and tofacitinib, compared with baricitinib and upadacitinib $(\mathrm{p}<0.001)$ (figure 4C).

For JAK1-independent pathways, filgotinib $200 \mathrm{mg}$ showed similar average daily inhibition of JAK2/TYK2-dependent G-CSF/pSTAT3 signalling as tofacitinib, but lower than baricitinib or upadacitinib (figure 4E). On the JAK2/JAK2-dependent GM-CSF/pSTAT5 pathway, upadacitinib and baricitinib showed $\geq 3$-fold greater inhibition than filgotinib $200 \mathrm{mg}(\mathrm{p}<0.001)$, with only upadacitinib achieving $>50 \%$ inhibition during the dose interval $(\mathrm{p}<0.001)$ (figure $4 \mathrm{~F})$. At clinical doses of upadacitinib, there were greater inhibition and time above $50 \%$ inhibition for JAK2-dependent and JAK3-dependent pathways signalling via G-CSF, GM-CSF, IFN $\gamma$ and IL-4 compared with filgotinib $200 \mathrm{mg}(\mathrm{p}<0.001)$ (figure 4C-F).

Taken together, these data demonstrate that filgotinib preferentially inhibits JAK1 pathways at therapeutic doses, with upadacitinib showing the least selectivity at clinical doses. As reported, baricitinib displays JAK1/JAK2 selectivity ${ }^{26}$ and tofacitinib mainly inhibits JAK1 and JAK3.

\section{In vitro study predictability and translation to clinical PD effects}

The in vitro predicted inhibition of IL-6/pSTAT1 and GM-CSF/ pSTAT5 in healthy donor blood was confirmed in ex vivo blood measurements from healthy volunteers orally dosed with filgotinib $200 \mathrm{mg}$. Average inhibitions of IL-6/pSTAT1 and GM-CSF/pSTAT5 with filgotinib were $78 \%$ and $36 \%$, respectively (figure 5A). Subtracting the placebo response, there was a good concordance between the measured IL-6 and GM-CSF daily inhibition (68\% and $15 \%$, respectively) to the in vitro predicted inhibitions (64\% and 6\%, respectively; figure 5A), directly demonstrating the clinical relevance of the WB cellular modelling calculations.
To determine if inhibition of cytokine responses in blood of healthy individuals was an appropriate surrogate for RA responses, we directly compared inhibition of cytokine pathways in a small number of blood samples of healthy and RA donors. Using these measured values, the predicted daily cytokine inhibition profile for IFN $\alpha /$ pSTAT5 in CD4 + cells by JAKinibs was comparable in healthy and RA blood (figure 5B). Across pathways, generally comparable average daily inhibitions were predicted for JAKinibs in healthy and RA blood (figure 5C), suggesting that measurements in healthy blood could be translated to predictive inhibitions in patients with RA.

\section{Pharmacological profiles of JAKinibs across cell populations and cytokine stimuli at clinical plasma exposures}

Clinical PD inhibition curves of cytokine signalling mediated by distinct JAK pairs were modelled (online supplemental figures 6-9). Average inhibition and time above 50\% inhibition are summarised at clinical doses (online supplemental table 7).

For JAK1/TYK2, IFN $\alpha /$ pSTAT5 signalling showed average inhibitions $>50 \%$ across all cell populations tested (figure 6A,B). Comparable levels of average daily inhibition were generally observed across cell populations, with upadacitinib showing greater inhibition in monocytes and CD4 + cells versus filgotinib $(p<0.05)$. The magnitude of inhibition was lower for IFN $\alpha$ driven pSTAT3 and pSTAT1, but all JAKinibs showed similar potency shifts. The weaker inhibition of IFN $\alpha /$ pSTAT1 $(<50 \%)$ by all JAKinibs potentially indicates more TYK 2 dependence for STAT1 phosphorylation. ${ }^{32}$

For JAK1/JAK2, IL-6/pSTAT1 signalling was inhibited by all JAKinibs, whereas there was half the inhibition of IL-6/ pSTAT3 (figure 6A,C). Differences in average daily inhibition by JAKinibs between cell types were observed, but overall filgotinib showed comparable or greater inhibition of IL-6/pSTAT1, while tofacitinib showed the least $(\mathrm{p}<0.001)$. In contrast, for the IFN $\gamma /$ pSTAT1 pathway (also mediated by JAK1/JAK2), upadacitinib and baricitinib showed greater inhibition of this pathway compared with filgotinib $(\mathrm{p}<0.001)$. There was a cell type-dependent difference in the magnitude of inhibition, with the greatest daily inhibition observed in B-cells and roughly half as much in neutrophils and monocytes, indicating a potentially greater reliance on JAK2 relative to JAK1 in mediating IFN $\gamma$ inhibition in specific cells.

For JAK1/JAK3, filgotinib consistently showed the least inhibition of common $\gamma$-chain cytokines (IL-2, IL-15 and IL-4) (figure 6A,D). Greater daily inhibition of IL-2-mediated and IL-15-mediated pSTAT5 followed tofacitinib > upadacitinib $>$ baricitinib $>$ filgotinib. There were significant differences between JAKinib daily inhibition that were dependent on cell type, but tofacitinib and upadacitinib reproducibly showed greater inhibition in $\mathrm{CD} 4+$ and CD $8+$ cells than filgotinib $(p<0.001)$. Tofacitinib, upadacitinib and baricitinib showed greater inhibition of IL-4/pSTAT5 than filgotinib in all cell types evaluated $(\mathrm{p}<0.05)$.

For JAK2/TYK2, the JAKinibs had a reduced effect on JAK2mediated signalling, with $<40 \%$ average inhibition of G-CSF/ pSTAT3 in monocytes and neutrophils and $<20 \%$ average inhibition of IL-12/pSTAT4 in NK cells and IL-23/pSTAT3 in CD8+ memoryT-cells, compared with the JAK1-mediated pathways (figure 6A,E). Upadacitinib and baricitinib showed significantly greater inhibition in monocytes compared with filgotinib. In the remaining cell types, inhibition by JAKinibs was approximately $25 \%-50 \%$ of that in monocytes, and a trend for lower inhibition by filgotinib compared with other JAKinibs was observed. 


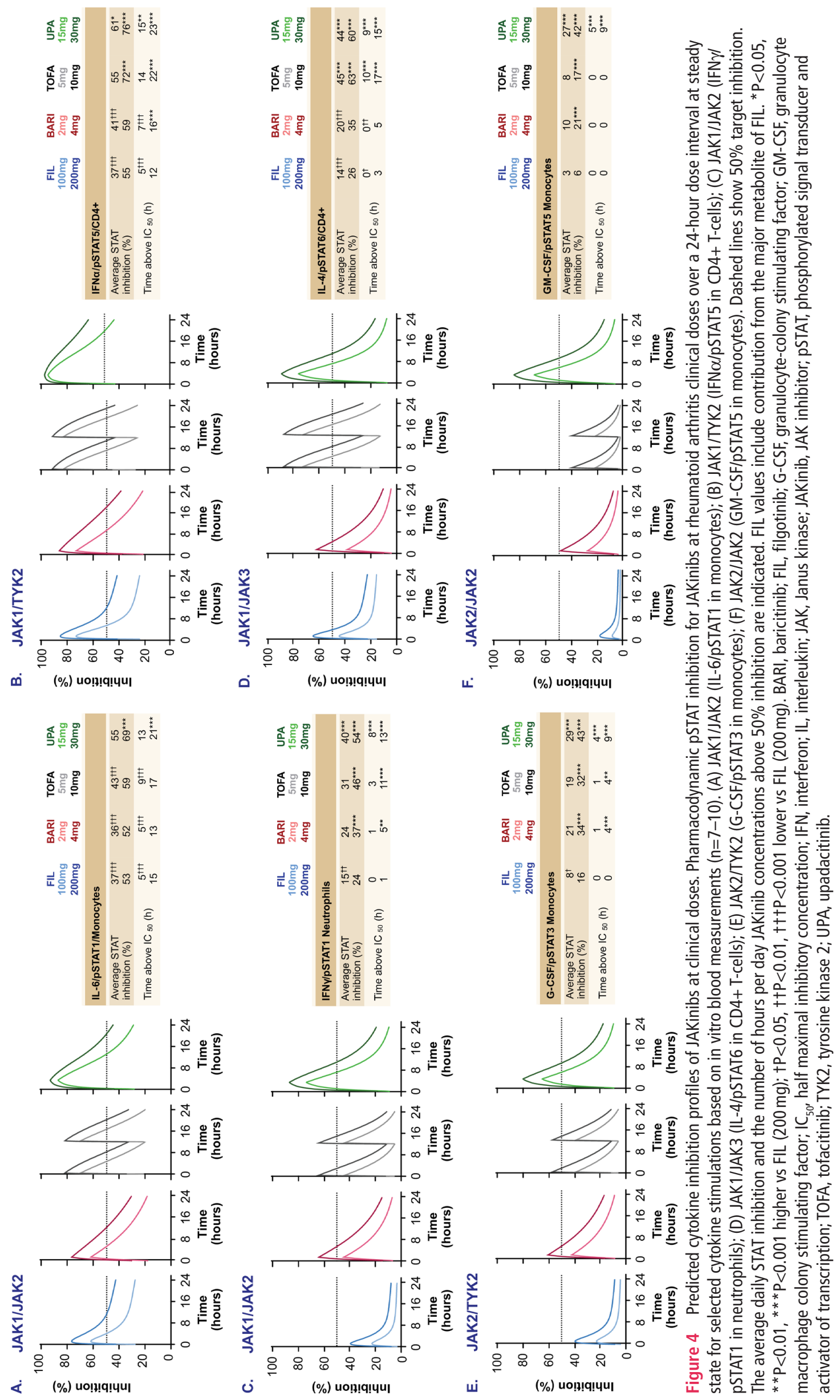




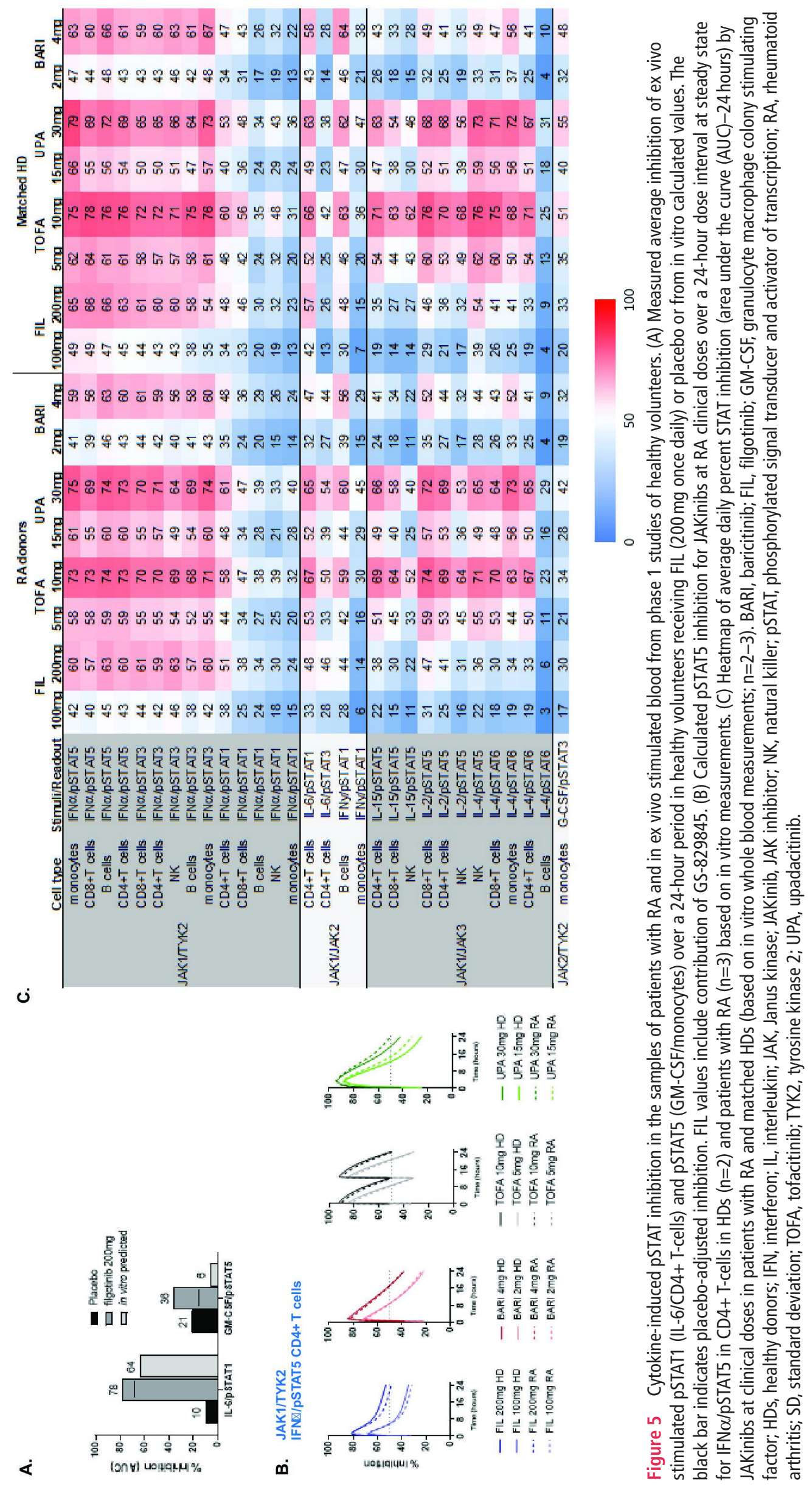



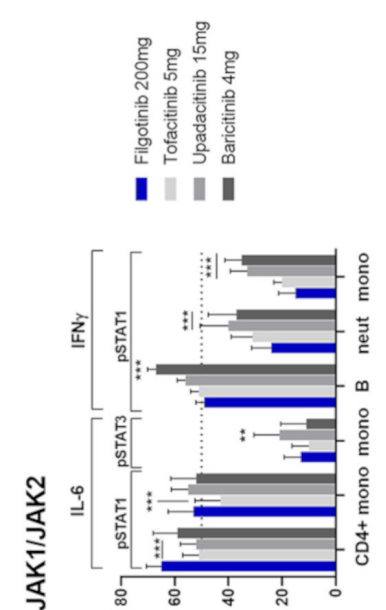

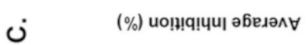

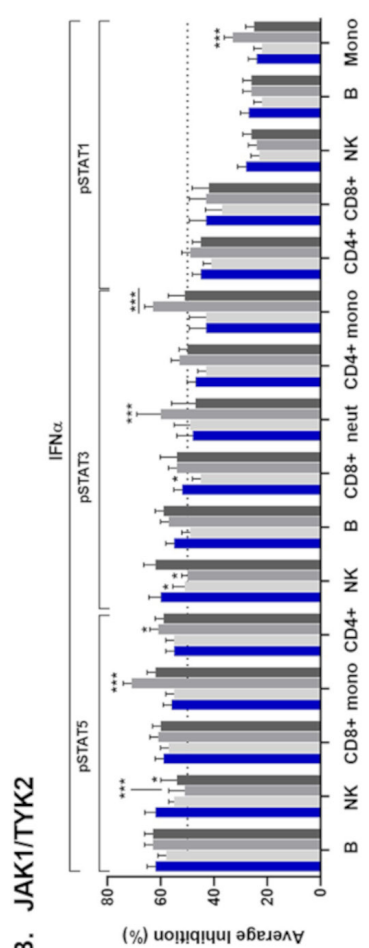

$\dot{0}$

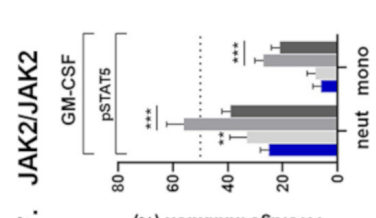

น்

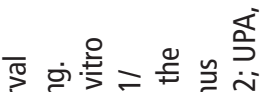

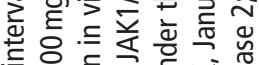

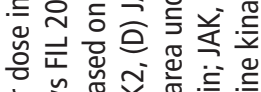

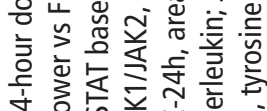

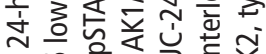

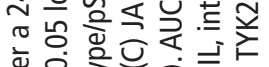
वे $\mathrm{v}=$

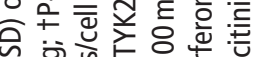

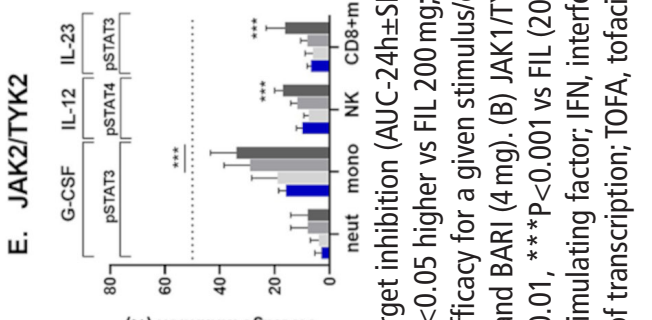

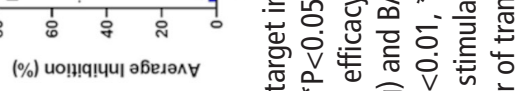

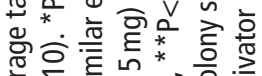

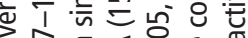
II

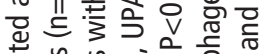

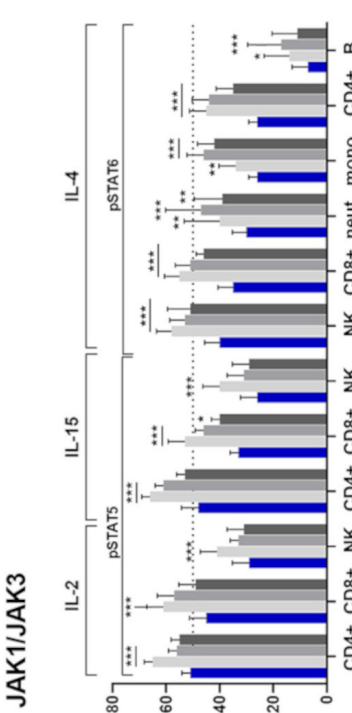

$\dot{0}$

(\%) ио!ı!q!чu әбеләл甘

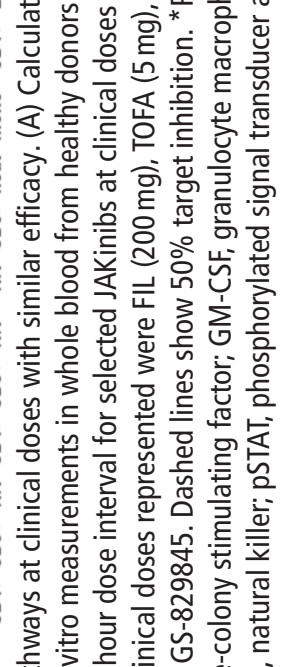

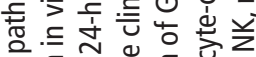

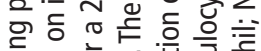

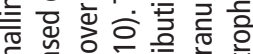

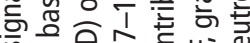

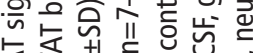

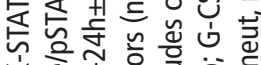

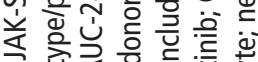

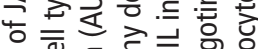

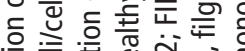

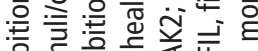

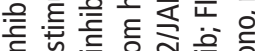

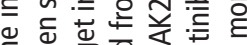

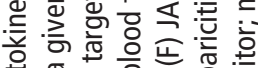
훙효

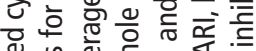

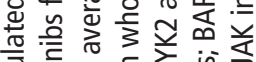
흥 ত্য

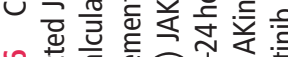

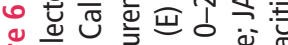

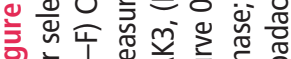


For JAK2/JAK2, inhibition of the GM-CSF/pSTAT5 pathway was significantly greater with tofacitinib, upadacitinib and baricitinib in neutrophils $(\mathrm{p}<0.01)$ and for upadacitinib and baricitinib in monocytes $(\mathrm{p}<0.001)$ compared with filgotinib (figure $6 \mathrm{~F}$ ). These data are consistent with the observation that upadacitinib and baricitinib inhibit JAK2 signalling at clinically relevant exposures, as seen for IFN $\gamma /$ pSTAT1 (figure $6 \mathrm{~A}, \mathrm{C}$ ) and G-CSF/pSTAT3 (figure 6A,E).

\section{DISCUSSION}

JAKinibs have similar efficacy in RA with distinct safety profiles, potentially reflecting differential JAK isoform selectivity. Enzymatically, JAKinibs have the greatest potency on JAK1, an isoform predominantly involved in inflammatory and innate immune responses. ${ }^{2122} 26-29$ Given that cytokine signalling involves JAK pairs, consideration solely of JAK1 enzymatic inhibition is insufficient to capture the differential effects of heterogeneous cytokine activation. Inhibition of JAK2 and JAK3 may contribute to AEs, given their involvement in regulating immune cell proliferation and homeostasis. In this study, we demonstrated that cytokine signals were differentially inhibited by filgotinib, tofacitinib, upadacitinib and baricitinib in human PBMCs and WB that translated to differential PD inhibition clinically, revealing unique JAKinib pharmacological selectivity profiles. Our key finding is that filgotinib inhibits JAK1-mediated IFN $\alpha$ and IL-6 similar to other JAKinibs at doses demonstrating similar efficacy, but exhibits reduced inhibition of JAK2-dependent and JAK3dependent pathways, potentially explaining the pharmacological basis for the reported differentiated safety profile.

In cellular assays, JAKinibs showed the greatest potency at inhibiting JAK1-dependent IFN $\alpha$ and IL-6 pathways and are calculated to have comparable average daily inhibition clinically, highlighting a shared JAK1 PD profile. These results are consistent with previously reported data for JAKinibs in PBMCs and blood. ${ }^{25} 27$ To our knowledge, this is the first comparison of JAKinib cytokine signalling inhibition of blood from healthy subjects and patients with RA, revealing an overall similar potency and predicted inhibition clinically across a panel of cytokines. Interestingly, IL-6/pSTAT3 inhibition in healthy donors was roughly half that of IL-6/pSTAT1, but in RA donors the predicted daily inhibition was comparable, indicating that some JAKinib responses in RA may not be adequately characterised using healthy donors as a surrogate. Additional studies would be required to verify these initial observations. As IL- 6 and IFN $\alpha$ are well-established drivers of autoimmunity, ${ }^{36-38}$ and IL-6 is a clinically validated target in RA, ${ }^{39}$ the observation that all JAKinibs strongly suppress IL- 6 and IFN $\alpha$ signalling with comparable potency implicates these pathways as primary drivers of efficacy in RA. ${ }^{25} 27$

In contrast to the comparable IFN $\alpha$ and IL- 6 inhibition, JAKinibs showed significant differences in other JAK1-dependent pathways, reflecting the interplay between cell type, JAK pairing and STAT substrate downstream of different cytokine stimuli. Nuances in cytokine inhibitory profiles for JAKinibs emerged. For example, filgotinib, baricitinib and upadacitinib showed more than threefold weaker inhibition of JAK1/JAK3-dependent IL-15/pSTAT5 pathway relative to IFN $\alpha / p S T A T 5$, whereas tofacitinib showed only a 1.8 -fold reduction. Filgotinib showed 6.9-fold weaker activity on JAK1/JAK2-dependent IFN $\gamma /$ pSTAT1 relative to IFN $\alpha /$ pSTAT5. At clinical exposures, these intrinsic potency differences were amplified. Consistently, there were lower average inhibition and less time above 50\% inhibition for filgotinib and baricitinib compared with tofacitinib and upadacitinib on JAK1/JAK3-dependent pathways. On the JAK1/ JAK2-dependent IFN $\gamma /$ pSTAT1 signalling, filgotinib showed lower inhibition than other JAKinibs in all cell types evaluated. This observation contrasts with the comparable inhibition on JAK1/JAK2-dependent IL-6/pSTAT1, indicating a stronger dependence of JAK1 in mediating IL-6/pSTAT1, as previously reported. ${ }^{640}$ Conversely, IFN $\gamma /$ pSTAT1 may be more reliant on JAK2, suggesting that, at clinical exposures, upadacitinib and baricitinib inhibit JAK2 more than filgotinib and tofacitinib. The lower inhibition of IFN $\gamma /$ pSTAT1 compared with IL-6/ pSTAT1 in CD14+ monocytes for JAKinibs is consistent with data reported by Dowty et al; ${ }^{25}$ however, in that study filgotinib showed comparable inhibition with other JAKinibs. This may be related to the use of a single average concentration to determine target coverage, as opposed to an integrated PD area under the curve approach, or use of saturating levels of cytokine stimulus. $^{25}$

JAK2-dependent cytokine signalling pathways were less inhibited than JAK1-dependent pathways for all JAKinibs, with filgotinib showing the least inhibition, including in neutrophils. The low level of inhibition of G-CSF/pSTAT3 in neutrophils is consistent with that observed by others in granulocytes. ${ }^{25} \mathrm{JAKi}-$ nibs are associated with serious infections, ${ }^{16-20}$ so minimising inhibition in neutrophils may maintain antipathogenic function. These data are consistent with the observation that filgotinib is the least potent compound for inhibition of IFN $\gamma / \mathrm{pSTAT} 1$, which is dependent on JAK2.

The data for JAKinib inhibition of JAK2-dependent and JAK3dependent cytokine responses corroborate previous findings that JAKinibs selectively inhibit cytokines that signal via JAK1 versus JAK2, and tofacitinib and upadacitinib have greater relative inhibition of JAK3-dependent common $\gamma$-chain cytokine receptor pathways compared with baricitinib or filgotinib at clinical doses. $^{242527}$ Furthermore, our data demonstrate that filgotinib has greater selectivity for cytokine signalling via JAK1 versus JAK2 than baricitinib and upadacitinib. The relatively low clinical inhibition of JAK2-dependent GM-CSF, IL-12, IL-23 and G-CSF is consistent with previous findings that filgotinib did not inhibit these pathways. ${ }^{25}$ Despite these shared findings, there were notable differences in reported results. Dowty et $a l^{25}$ claim 'limited differentiation' of JAKinib selectivity; however, it was not statistically powered for comparison, nor did they provide a clinical PD profile of cytokine inhibition. Their approach used an average clinical JAKinib concentration at similarly efficacious therapeutic doses. ${ }^{25}$ As inhibitory activity is not linear over a concentration range, methodology using a single average concentration may minimise PD differences, potentially obscuring clinical safety and efficacy implications. Currently, it is unknown what level of target inhibition or time above 50\% inhibition could have clinical impact on safety, and subtle differences may be meaningful.

Our data indicate that JAKinibs have clinically differentiated PD profiles that mechanistically may underlie incidence rates of presumed class-effect AEs. ${ }^{41-48}$ Selectivity for JAK1 can drive RA efficacy, whereas increasing inhibition of JAK2-dependent and JAK3-dependent pathways may elicit safety liabilities. This is predicted from genetic deletion and clinical data, showing that JAK2 is essential for erythropoiesis, myelopoiesis and platelet production, and JAK3 is critical for lymphocyte proliferation and homeostasis. ${ }^{49}$ Compared with other JAKinibs, filgotinib had less inhibition of JAK1-mediated IFN $\gamma$ (JAK1/JAK2) and IL-2, IL-15 and IL-4 (JAK1/JAK3), JAK2-mediated G-CSF, IL-12 and IL-23 (JAK2/TYK2), and GM-CSF (JAK2/JAK2). The reduced inhibition of JAK2-dependent and JAK3-dependent 
cytokine signalling by filgotinib may underlie its lower reported impact on homeostatic immune functions that control NK cells, platelet numbers, anaemia, lymphocyte numbers, infection and HZ (online supplemental table 1). ${ }^{49-51}$

This study has limitations. We established that JAKinib effects are context-dependent and influenced by parameters including cytokine stimulus, STAT substrate and cell type. The interdependence of these parameters needs to be considered when extrapolating these data. We evaluated cytokines that signal via distinct JAK pairs and generalised the results to other cytokines using the same JAK pairs. Our results, however, indicate that the inhibitory effect of each JAKinib is dependent on the specific cytokine stimulus, STAT substrate and cell type, so the association of pathway inhibition with clinical impact would require cellspecific cytokine evaluation. To validate whether the findings in healthy controls would predict cellular effects at clinical exposures, we measured ex vivo effects of IL-6/pSTAT1 and GM-CSF/ pSTAT5 in healthy volunteers dosed with filgotinib and showed good agreement with the in vitro-derived extent of inhibition. Although we studied JAKinib cytokine inhibition in blood from patients with RA, the number of donors and the breadth of the cytokine panel were limited. The validity of extrapolating these findings to a broad spectrum of cytokine responses in patients with RA requires further evaluation. While this study provides a potential mechanistic basis for the differential JAKinib rates of AEs from meta-analyses, confirmation would involve head-tohead clinical evaluations.

In conclusion, we demonstrated that, based on in vitro cellular assays and clinical pharmacokinetics, filgotinib is predicted to have a differentiated cytokine PD profile in the clinical setting compared with other JAKinibs. JAK1-dependent IL-6 and IFN $\alpha$ pathways were comparably inhibited by JAKinib doses that have similar efficacy in RA, but filgotinib demonstrated reduced JAK2 and JAK3 activity, providing a potential mechanistic basis for the reported differences in its safety profiles.

Acknowledgements We extend our thanks to the patients and their families. We wish to acknowledge Derek Stonich for his assistance with data management and IT support. Research support was provided by Primity Bio. Writing and editorial support was provided by Impact Communication Partners, Inc.

Contributors PGT and JAD designed the research. PGT, JAD and FC analysed the data and performed statistical analyses. BM, RG and AM analysed clinical PK and PD data. All authors approved the final manuscript.

Funding This study was funded by Gilead Sciences, Inc.

Competing interests PGT, BM, FC, AM and JAD are employees of Gilead Sciences, Inc. RG is an employee of Galapagos SASU.

Patient consent for publication Not required.

Ethics approval The study was conducted in accordance with the ethical principles of the Declaration of Helsinki and Good Clinical Practice guidelines, and was approved by the Independent Ethics Committee or Institutional Review Board. The study was performed in compliance with the ethical principles of good clinical practice and according to the ICH Harmonised Tripartite Guideline. Patients provided written informed consent to participate in the study and had the right to withdraw at any time.

Provenance and peer review Not commissioned; externally peer reviewed.

Data availability statement All data relevant to the study are included in the article or uploaded as supplementary information. Anonymised individual patient data will be available upon request to qualified external researchers 6 months after FDA and European Medicines Agency approval per Gilead's Clinical Trial Disclosure and Data Transparency Policy as posted at https://www.gilead.com/research/ disclosure-and-transparency.

Supplemental material This content has been supplied by the author(s). It has not been vetted by BMJ Publishing Group Limited (BMJ) and may not have been peer-reviewed. Any opinions or recommendations discussed are solely those of the author(s) and are not endorsed by BMJ. BMJ disclaims all liability and responsibility arising from any reliance placed on the content. Where the content includes any translated material, BMJ does not warrant the accuracy and reliability of the translations (including but not limited to local regulations, clinical guidelines, terminology, drug names and drug dosages), and is not responsible for any error and/or omissions arising from translation and adaptation or otherwise.

Open access This is an open access article distributed in accordance with the Creative Commons Attribution Non Commercial (CC BY-NC 4.0) license, which permits others to distribute, remix, adapt, build upon this work non-commercially, and license their derivative works on different terms, provided the original work is properly cited, appropriate credit is given, any changes made indicated, and the use is non-commercial. See: http://creativecommons.org/licenses/by-nc/4.0/.

\section{REFERENCES}

1 Gadina M, Le MT, Schwartz DM. Janus kinases to jakinibs: from basic insights to clinical practice. Rheumatology 2019;58:i4-16.

2 Morris R, Kershaw NJ, Babon JJ. The molecular details of cytokine signaling via the JAK/STAT pathway. Protein Sci 2018;27:1984-2009.

3 O'Shea JJ, Murray PJ. Cytokine signaling modules in inflammatory responses. Immunity 2008;28:477-87.

4 Imada K, Leonard WJ. The JAK-STAT pathway. Mol Immunol 2000;37:1-11.

5 Neubauer H, Cumano A, Müller M, et al. Jak2 deficiency defines an essential developmental checkpoint in definitive hematopoiesis. Cell 1998:93:397-409.

6 O'Shea JJ, Kontzias A, Yamaoka K, et al. Janus kinase inhibitors in autoimmune diseases. Ann Rheum Dis 2013;72 Suppl 2:ii111-5.

7 Park SO, Wamsley HL, Bae K, et al. Conditional deletion of JAK2 reveals an essential role in hematopoiesis throughout mouse ontogeny: implications for JAK2 inhibition in humans. PLoS One 2013:8:e59675.

8 Vainchenker W, Constantinescu SN. Jak/Stat signaling in hematological malignancies. Oncogene 2013;32:2601-13.

9 DiSanto JP, Müller W, Guy-Grand D, et al. Lymphoid development in mice with a targeted deletion of the interleukin 2 receptor gamma chain. Proc Natl Acad Sci U S A 1995:92:377-81.

10 Lin J-X, Leonard WJ. The common cytokine receptor $\gamma$ chain family of cytokines. Cold Spring Harb Perspect Biol 2018;10:a028449.

11 Notarangelo LD, Giliani S, Mazza C, et al. Of genes and phenotypes: the immunological and molecular spectrum of combined immune deficiency. Defects of the gamma(c)-JAK3 signaling pathway as a model. Immunol Rev 2000;178:39-48.

12 Rodig S, Kaplan D, Shankaran V, et al. Signaling and signaling dysfunction through the interferon gamma receptor. Eur Cytokine Netw 1998;9:49-53.

13 Suzuki K, Nakajima H, Saito Y, et al. Janus kinase 3 (Jak3) is essential for common cytokine receptor gamma chain (gamma(c))-dependent signaling: comparative analysis of gamma(c), Jak3, and gamma(c) and Jak3 double-deficient mice. Int Immunol 2000;12:123-32.

14 Thomis DC, Gurniak CB, Tivol E, et al. Defects in B lymphocyte maturation and T lymphocyte activation in mice lacking Jak3. Science 1995;270:794-7.

15 O'Shea JJ, Schwartz DM, Villarino AV, et al. The JAK-STAT pathway: impact on human disease and therapeutic intervention. Annu Rev Med 2015:66:311-28.

16 Olumiant (baricitinib) prescribing information. Available: http://pi.lilly.com/us/ olumiant-uspi.pdf [Accessed 12 May 2020].

17 Xeljanz (tofacitinib) European public assessment report. Available: https://www.ema. europa.eu/en/documents/product-information/xeljanz-epar-product-information_en. pdf [Accessed 12 May 2020].

18 Xeljanz (tofacitinib) prescribing information. Available: http://labeling.pfizer.com/ ShowLabeling.aspx?id=959 [Accessed 15 May 2020].

19 Rinvoq (upadacitinib) prescribing information. Available: https://www.rinvoq.com/? cid=ppc_ppd_ggl_branded_general_rinvoq_prescribing_information_exact_ usrnqr190596 [Accessed 12 May 2020]

20 Genovese MC, Kalunian K, Gottenberg J-E, et al. Effect of filgotinib vs placebo on clinical response in patients with moderate to severe rheumatoid arthritis refractory to disease-modifying antirheumatic drug therapy: the finch 2 randomized clinical trial. JAMA 2019;322:315-25.

21 Van Rompaey L, Galien R, van der Aar EM, et al. Preclinical characterization of GLPG0634, a selective inhibitor of JAK1, for the treatment of inflammatory diseases. $J$ Immunol 2013:191:3568-77.

22 Galien R, Vayssière B, De Vos S, eds. Analysis of the JAK1 selectivity of GLPG0634 and its main metabolite in different species, healthy volunteers and rheumatoid arthritis patients. Abstract 478. American College of Rheumatology and Association of Rheumatology Health Professionals annual meeting. San Diego, California, 2013

23 Cox L, Cools J. Jak3 specific kinase inhibitors: when specificity is not enough. Chem Biol 2011;18:277-8.

24 Haan C, Rolvering C, Raulf F, et al. Jak1 has a dominant role over Jak3 in signal transduction through $\gamma c$-containing cytokine receptors. Chem Biol 2011;18:314-23.

25 Dowty ME, Lin TH, Jesson Ml, et al. Janus kinase inhibitors for the treatment of rheumatoid arthritis demonstrate similar profiles of in vitro cytokine receptor inhibition. Pharmacol Res Perspect 2019;7:e00537.

26 Fridman JS, Scherle PA, Collins R, et al. Selective inhibition of JAK1 and JAK2 is efficacious in rodent models of arthritis: preclinical characterization of INCB028050. J Immunol 2010;184:5298-307. 
27 Mclnnes IB, Byers NL, Higgs RE, et al. Comparison of baricitinib, upadacitinib, and tofacitinib mediated regulation of cytokine signaling in human leukocyte subpopulations. Arthritis Res Ther 2019;21:183.

28 Meyer DM, Jesson MI, Li X, et al. Anti-Inflammatory activity and neutrophil reductions mediated by the JAK1/JAK3 inhibitor, CP-690,550, in rat adjuvant-induced arthritis. J Inflamm 2010;7:41.

29 Parmentier JM, Voss J, Graff C, et al. In vitro and in vivo characterization of the JAK1 selectivity of upadacitinib (ABT-494). BMC Rheumatol 2018;2:23.

30 Leonard WJ, O'Shea JJ. Jaks and STATs: biological implications. Annu Rev Immunol 1998;16:293-322.

31 Harigai M, Honda S. Selectivity of Janus kinase inhibitors in rheumatoid arthritis and other immune-mediated inflammatory diseases: is expectation the root of all headache? Drugs 2020;80:1183-201.

32 Burke JR, Cheng L, Gillooly KM, et al. Autoimmune pathways in mice and humans are blocked by pharmacological stabilization of the Tyk2 pseudokinase domain. Sci Trans/ Med 2019;11:eaaw1736.

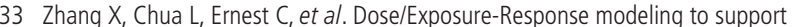
dosing recommendation for phase III development of Baricitinib in patients with rheumatoid arthritis. CPT Pharmacometrics Syst Pharmacol 2017;6:804-13.

34 Center for Drug Evaluation and Research (CDER). Application number: 2032140rig1s000. NDA 203214: tofacitinib. Available: https://www.accessdata.fda. gov/drugsatfda_docs/nda/2012/2032140rig1s000ClinPharmR.pdf [Accessed 19 May 2020].

35 Klünder B, Mittapalli RK, Mohamed M-EF, et al. Population pharmacokinetics of upadacitinib using the immediate-release and extended-release formulations in healthy subjects and subjects with rheumatoid arthritis: analyses of phase I-III clinical trials. Clin Pharmacokinet 2019;58:1045-58.

36 Alghasham A, Rasheed Z. Therapeutic targets for rheumatoid arthritis: progress and promises. Autoimmunity 2014;47:77-94.

37 Conigliaro P, Perricone C, Benson RA, et al. The type I IFN system in rheumatoid arthritis. Autoimmunity 2010;43:220-5.

38 Crow MK. Type I interferon in organ-targeted autoimmune and inflammatory diseases. Arthritis Res Ther 2010;12 Suppl 1:S5.

39 Yoshida Y, Tanaka T. Interleukin 6 and rheumatoid arthritis. Biomed Res Int 2014:2014:698313

40 Heinrich PC, Behrmann I, Müller-Newen G, et al. Interleukin-6-Type cytokine signalling through the gp130/Jak/STAT pathway. Biochem J 1998;334 (Pt 2:297-314.
41 Fleischmann R, Pangan AL, Song I-H, et al. Upadacitinib versus placebo or adalimumab in patients with rheumatoid arthritis and an inadequate response to methotrexate: results of a phase III, double-blind, randomized controlled trial. Arthritis Rheumatol 2019;71:1788-800.

42 Genovese MC, Kremer J, Zamani 0, et al. Baricitinib in patients with refractory rheumatoid arthritis. N Eng/ J Med 2016:374:1243-52.

43 Kavanaugh A, Kremer J, Ponce L, et al. Filgotinib (GLPG0634/GS-6034), an oral selective JAK1 inhibitor, is effective as monotherapy in patients with active rheumatoid arthritis: results from a randomised, dose-finding study (Darwin 2). Ann Rheum Dis 2017;76:1009-19.

44 Smolen JS, Pangan AL, Emery P, et al. Upadacitinib as monotherapy in patients with active rheumatoid arthritis and inadequate response to methotrexate (SELECTMONOTHERAPY): a randomised, placebo-controlled, double-blind phase 3 study. Lancet 2019;393:2303-11.

45 Taylor PC, Keystone EC, van der Heijde D, et al. Baricitinib versus placebo or adalimumab in rheumatoid arthritis. N Eng/ J Med 2017;376:652-62.

46 Westhovens R, Taylor PC, Alten R, et al. Filgotinib (GLPG0634/GS-6034), an oral JAK1 selective inhibitor, is effective in combination with methotrexate (MTX) in patients with active rheumatoid arthritis and insufficient response to MTX: results from a randomised, dose-finding study (Darwin 1). Ann Rheum Dis 2017;76:998-1008.

47 Winthrop KL, Genovese M, Combe B, eds. Pooled safety analyses from phase 3 studies of filgotinib in patients with rheumatoid arthritis. Available at. Atlanta, GA: American College of Rheumatology annual meeting, 2019. https://acrabstracts.org/ abstract/pooled-safety-analyses-from-phase-3-studies-of-filgotinib-in-patients-withrheumatoid-arthritis/

48 Wollenhaupt J, Lee E-B, Curtis JR, et al. Safety and efficacy of tofacitinib for up to 9.5 years in the treatment of rheumatoid arthritis: final results of a global, open-label, long-term extension study. Arthritis Res Ther 2019;21:89.

49 Winthrop KL. The emerging safety profile of JAK inhibitors in rheumatic disease. Nat Rev Rheumatol 2017;13:234-43.

50 Combe B, Kivits A, Tanaka Y, et al. Efficacy and safety of filgotinib for patients with rheumatoid arthritis with inadequate response to methotrexate: Finch1 primary outcome results. Abstract LB0001. Madrid, Spain: European League Against Rheumatism, 2019.

51 Westhovens RRW, van der Heijde D, Ching D, et al. Efficacy and safety of filgotinib for patients with rheumatoid arthritis naive to methotrexate therapy: FINCH3 primary outcome results [abstract]. Arthritis Rheumatol 2019;71. 